\title{
SEPARABLE DIOPHANTINE EQUATIONS
}

BY

\author{
E. T. BELL
}

Theoretically, as noted by Skolem [3, p. 2] $\left(^{1}\right)$, the general problem of algebraic diophantine analysis is reducible to the case in which occur only equations and inequalities of degree not higher than the second. For the extensive class of separable systems defined in $\$ 6$, this reduction can be performed effectively, eventuating in the complete integer solutions of the equations concerned. The general method is strictly elementary, but none the less powerful within its natural range on that account. Among the more immediate applications are complete solutions of certain types of homogeneous equations of the second degree, only special cases of which have been solved hitherto by advanced methods, including that of generalized quaternions. The equation $x_{1}^{2}+\cdots+x_{n}^{2}=y^{2}$, for example, does not seem to be adapted to such methods, as a sum of $n$ squares is not factorable, for all $n$, in a ring.

For simplicity of statement only, the method is presented for the domain of rational integers. A few slight and obvious verbal changes suffice to extend the entire discussion to any unique factorization domain, in particular to domains in which there is a Euclidean algorithm. The extension to principal ideal rings of algebraic integers, for instance, yields results of interest in the domain of rational integers.

\section{Extended multiplicative EQUations}

1. Extended and simple systems. With the exception of the symbol $\sum$ of summation, all letters throughout the paper denote rational integers.

A monomial $x^{a} y^{b} \cdots z^{c}$ in which $x, y, \cdots, z$ are independent variables (or independent unknowns), and $a, b, \cdots, c$ are constants greater than 0 , is elementary if at least one of $a, b, \cdots, c$ is restricted to be 1 and $a+b$ $+\cdots+c>1$.

Whether elementary or unrestricted, the monomials in a set are independent if no two of them have a variable in common.

An equation of the type

$$
m_{1} X_{1}+\cdots+m_{n} X_{n}=0
$$

in which $n>2, m_{1}, \cdots, m_{n}$ are constants all different from zero, and $X_{1}, \cdots, X_{n}$ are independent elementary monomials, is an extended multiplicative equation.

Two extended multiplicative equations are connected if they have in com-

Presented to the Society, August 14, 1944; received by the editors April 20, 1944.

( $\left.{ }^{1}\right)$ Numbers in brackets refer to the references cited at the end of the paper. 
mon at least one variable. If each pair of a set of equations is connected, the set is connected. A single extended multiplicative equation, or a connected set, is an extended system.

The most important detail implicit in the last definition is that all the monomials in an extended system are elementary.

A set of equations of the type

$$
c_{1} Y_{1}=c_{2} Y_{2}=\cdots=c_{8} Y_{8},
$$

in which $s \geqq 2, c_{1}, \cdots, c_{s}$ are constants all different from zero, and $Y_{1}, \cdots, Y_{s}$ are independent monomials, is a simple multiplicative set.

Two simple multiplicative sets are connected if they have in common at least one variable. If each pair of two or more sets is connected, or if there is but one set, the totality of sets constitute a simple system.

An extended system is thus more general than a simple system in that the equations of the extended system are not restricted, as in the simple system, to two terms. A simple system is more general than an extended system in that the monomials in the simple system are not restricted, as in the extended system, to be elementary.

In the method for extended systems developed here, the known theory of simple systems $[1 ; 4]$ is a prerequisite. Acquaintance with algoristic details of that theory, however, is not necessary for following the applications to the solution of extended systems, as enough of the technique is recalled incidentally in the next four sections.

2. Expansion and contraction. The monomial $x^{a} y^{b} \cdots z^{c}$ is expanded with respect to any one of its variables $x, y, \cdots, z$, say $z$, if $z^{c}$ is replaced by the product of $c$ variables, say $z_{1}, \cdots, z_{c}$, all distinct from one another and from the remaining variables $x, y, \cdots$ in the given monomial. Thus the expansion with respect to $z$ is $x^{a} y^{b} \cdots z_{1} \cdots z_{c}$ with $x, y, \cdots, z_{1}, \cdots, z_{c}$ all distinct. The total expansion

$$
x_{1} \cdots x_{a} y_{1} \cdots y_{b} \cdots z_{1} \cdots z_{c}
$$

is obtained by expanding with respect to each variable in turn. A totally expanded monomial of degree $n$ is thus a product of first powers of $n$ independent variables.

The inverse of expansion is contraction. A monomial is contracted with respect to any subset (proper or improper) of its variables by replacing each of the variables in the subset by the same variable, the new variable being distinct from all those not replaced. The total contraction is the result of replacing each of the variables in the monomial by the same variable. Thus the total contraction of $x^{a} y^{b} \cdots z^{c}$ is $w^{d}$, where $d=a+b+\cdots+c$.

In solving simple systems, at least one of the monomials in the system is totally expanded, and the system thus modified is solved. To the solution of the modified system are adjoined the equations giving the contractions neces- 
sary to reproduce the original system. The modified system together with the equations of contraction constitute a new system. By repeated expansions and contractions the solution of the original system is obtained.

3. Free and bound parameters. A variable whose range of values is the integers is a free parameter.

Distinct variables $\xi, \eta, \cdots, \zeta$ which may take only such values as satisfy a binding equation of the form

$$
m \xi^{a} \eta^{b} \cdots \zeta^{c}=n,
$$

where $m, a, b, \cdots, c, n$ are constant integers and $m n \neq 0, a, b, \cdots, c>0$, are bound parameters.

The solution of the indicated binding equation is equivalent to that of an associated system of linear diophantine equations. Let $p_{1}, \cdots, p_{[n]}$ be all the distinct positive prime divisors of $n$, and for any integer $s$, let $p_{j}^{s_{j}}, s_{j} \geqq 0$, be the highest power of $p_{j}$ dividing $s$. By comparison of exponents of $p_{j}$ in the binding equation, the associated linear system is

$$
\begin{gathered}
m_{j}+a \xi_{j}+b \eta_{j}+\cdots+c \zeta_{j}=n_{j}, \\
\xi_{i} \geqq 0, \eta_{j} \geqq 0, \cdots, \zeta_{j} \geqq 0, \quad j=1, \cdots,[n] .
\end{gathered}
$$

There are no solutions if the G.C.D. of $m_{j}, a, b, \cdots, c$ does not divide $n_{j}$ (all $j$ ), or if for at least one $j, m_{j}>n_{j}$. If $|m|=1$, and $\xi^{a} \eta^{b} \cdots \zeta^{c}$ is elementary, the system has solutions. If there are solutions, there are only a finite number, $n^{\prime}$, say

$$
\left(\xi_{j}, \eta_{j}, \cdots, \zeta_{j}\right)=\left(\xi_{j i}, \eta_{j i}, \cdots, \zeta_{j i}\right), \quad i=1, \cdots, n^{\prime} .
$$

Let each of $e_{\xi}, e_{\eta}, \cdots, e_{\xi}$ be a definite one of $1,-1$. All solutions of the binding equation are

$$
(\xi, \eta, \cdots, \zeta)=\left(e_{\xi} p_{j}^{\xi_{j i}}, e_{\eta} p_{j}^{\eta_{j i}}, \cdots, e_{\zeta} p_{j}^{\xi_{j i}}\right),
$$

where the repeated index $j$ indicates a product over $j=1, \cdots,[n]$, and the units are assigned so that $e_{\xi}^{a} e_{\eta}^{b} \cdots e_{\zeta}^{c}=1$ or -1 according as $m n>0$ or $m n<0$.

The detail of importance for extended systems is that the binding equation has solutions if $|m|=1$ and $\xi^{a} \eta^{b} \ldots \zeta^{c}$ is elementary. The solution of a system of two or more binding equations is an immediate extension of the procedure for a single equation.

Binding equations arise as follows in solving either simple or extended systems. Constant coefficients $l, m, \cdots$ other than $1,-1$ are replaced by variables $\lambda, \mu, \cdots$ respectively, distinct among themselves and all distinct from the original variables in the system. Thus modified, the system has only unit coefficients. If a parametric solution of the modified system presents $\lambda, \mu, \cdots$ in the form $\lambda=l^{\prime} L, \mu=m^{\prime} M, \cdots$, where $l^{\prime}, m^{\prime}, \ldots$ are constants and $L, M, \cdots$ are monomials in the parameters, the binding equations are $l^{\prime} L=l, m^{\prime} M=m, \cdots$.

The concept of binding equations can be extended to equations of the type 


$$
m_{1} X_{1}+\cdots+m_{s} X_{s}=n,
$$

where $m_{1}, \cdots, m_{s}, n$ are constants not equal to 0 , and $X_{1}, \cdots, X_{s}$ are monomials, not necessarily independent.

4. Reducibility. Each equation in a simple system has only two terms. An equation in more than two terms is reducible if it can be derived from a twoterm equation by polynomial replacements of the variables in the two-term equation. A system of equations is reducible if each equation of the system in more than two terms is reducible. Unless otherwise noted, the coefficients in the substituted polynomials are restricted to be rational numbers, although this is not necessary; the discussion is almost the same if the coefficients are elements of the quotient field of any unique factorization domain.

Reducibility cuts across the order (number of terms) and the degree of equations and, where applicable, either provides a means of solving the equations or isolates their essential difficulties. It also unites apparently unrelated equations in classes according to the simple systems from which they are derivable, and makes possible a unified treatment of all.

5. Examples for \$4. The arrow, $\rightarrow$, is to be read "is replaced by"; $(a, b, \cdots)$ denotes as usual the G.C.D. of $a, b, \cdots$ "Solution," as always, means "complete integer solution."

The solution of $\xi \eta=\zeta \phi$ is

$$
\xi=a b, \quad \eta=c d, \quad \zeta=a d, \quad \phi=c b,
$$

$a, b, c, d$ free parameters. If the parameters are restricted by the G.C.D. condition $(b, d)=1$, the solution contains no duplications. The process of solving any simple system [1] automatically furnishes a set of G.C.D. conditions which, imposed, exclude duplications. These need not be stated if the objective, as here, is the solution.

Contraction with respect to $\zeta, \phi$ adjoins the new equation $a d=b c$, of the same type as the original; whence there is the solution of

$$
\begin{gathered}
\xi \eta=\zeta^{2}: \\
\xi=f g^{2}, \quad \eta=f h^{2}, \quad \zeta=f g h, \quad(g, h)=1 .
\end{gathered}
$$

Of the infinity of equations reducible to the last, the following will suffice to illustrate reducibility:

$$
\begin{aligned}
x^{2}+y^{2} & =z^{2} ; \\
x y+y z+z x & =0 ; \\
x^{2}+y^{4} & =z^{2} .
\end{aligned}
$$

The first is derived by $\xi \rightarrow z+x, \eta \rightarrow z-x, \zeta \rightarrow y$. The solution is therefore given by $2 x=f\left(g^{2}-h^{2}\right), y=f g h, 2 z=f\left(g^{2}+h^{2}\right)$; whence the usual solution follows by considering the possible forms of $f, g, h$ modulo 2 . This equation is the total contraction of its total expansion 


$$
x_{1} x_{2}+y_{1} y_{2}=z_{1} z_{2},
$$

which is solvable. But for reasons which will appear in the discussion of separable equations, the solution of the totally contracted equation is not obtainable from that of its total expansion.

The second equation is derived by $\xi \rightarrow x+y, \eta \rightarrow x+z, \zeta \rightarrow x$; the solution is therefore

$$
x=f g h, \quad y=f g(g-h), \quad z=f h(h-g) .
$$

The solution of the third equation is obtained from that of the second by applying the solution of the simple equation $f g h=k^{2}: f=c_{1}^{2} c_{4} c_{5} c_{7} c_{9}, g=c_{2}^{2} c_{5} c_{6} c_{7} c_{8}$, $h=c_{3}^{2} c_{4} c_{6} c_{8} c_{9}, k=c_{1} c_{2} c_{3} c_{4} c_{5} c_{6} c_{7} c_{8} c_{9}$, in 9 free parameters $c_{j}$.

It may be recalled that either of the methods $[1 ; 4]$ for obtaining the solution of a simple system $S$ produces the solution in terms of a certain number $N(S)$, characteristic of $S$, of parameters both necessary and sufficient for the solution. For $S \equiv f g h=k^{2}, N(S)=9$, as above. For

$$
S \equiv x^{9}=y^{5}=u^{4} v^{4}=\text { wrst, }
$$

all the variables being independent, $N(S)=46,217,626$. Equally rudimentary systems for which $N(S)$ exceeds any preassigned number are readily constructed. Contraction increases $N(S)$. Thus for the totally expanded form $S^{\prime}$ of the last system, $N\left(S^{\prime}\right)=1440$; and generally (a frequently occurring case), for

$$
S \equiv x_{1} \cdots x_{a}=y_{1} \cdots y_{b}=\cdots=w_{1} \cdots w_{c},
$$

the $a+b+\cdots+c$ variables being independent, $N(S)=a b \cdots c$.

A point of some interest may be noted in passing. Geometrically, a simple system $S$ defines a rational variety $V$ in space of two or more dimensions, and $V$ is parametrized by a certain number $n(V)$ of parameters. It is observed that $N(S) / n(V)$ tends to infinity more rapidly than any positive integral power of $m v d$, where $m$ is the number of monomials, $v$ the number of independent variables, and $d$ is the highest degree of any monomial in $S$. It is not clear why specifying the integer points on $V$ should so greatly increase the necessary and sufficient number of parameters. Libri [2] insisted that an algebraic diophantine system is "not indeterminate, ..., but overdetermined"; it is implicitly transcendental and should be extended by conditional equations (in circular functions) to exclude all but integer values of the variables. This proposal was rejected by Dirichlet as a valid but trivial heresy, and it has not been adopted. In any case it fails to yield the correct value for any $N(S)$.

To illustrate bound parameters,

$$
m x^{2}+n y^{2}=n z^{2},
$$

with $m, n$ constants not equal to 0 , is derived from $\mu \xi^{2}=\nu \eta \zeta$, in 5 variables, 
whose solution is

$$
\begin{gathered}
\mu=g m_{1} m_{2}, \quad \xi=n_{1} n_{2} n_{3} n_{4} n_{5} \theta \phi \psi, \\
\nu=g n_{1} n_{2} n_{3} n_{4} n_{5}^{2}, \quad \eta=m_{1} n_{1} n_{3} \theta \phi^{2}, \quad \zeta=m_{2} n_{2} n_{4} \theta \psi^{2},
\end{gathered}
$$

in 11 free parameters. The required solution is derived from this by

$$
\mu \rightarrow m, \quad \nu \rightarrow n, \quad \xi \rightarrow x, \quad \eta \rightarrow z+y, \quad \zeta \rightarrow z-y,
$$

so that now only $\theta, \phi, \psi$ are free.

6. Separability. This concerns the sense in which "solution" is to be understood in the applications considered in later sections. The sense is a customary one; but for definiteness it is stated here in the form given by Skolem [2, p. 2]: "Im Falle unendlich vieler Lösungen sollen diese durch ein allgemeine Formel dargestellt werden, oder man soll ein Verfahren angeben, mit dessen Hilfe man alle Lösungen allmählich finden kann."

"Resolution" is used to avoid confusion between "solution" as "process of solving" and "solution" as "result of solving": a system is "resolved" to give its "solution." Equivalent resolutions are different processes of solving a system; they necessarily produce the same solution. The "Verfahren" is the resolution; the "Lösungen" the solution, meaning, as always, "complete solution." All resolutions of a given system are equivalent. It therefore suffices to produce one, if general formulas for the solution are not forthcoming.

A system $S$ is separable if a resolution of $S$ is equivalent to one or more of the following:

(A) the resolution of independent extended equations;

(B) the resolution of simple systems;

(C) the resolution of systems of linear diophantine equations, the total number of systems in (A), (B), (C) being finite.

It will be shown in the next section that there is a finite process for (A). Since finite processes are available for (B), (C), a separable system is resolvable and has a solution in the senses defined. It is to be noticed that the equations in (A) are independent. Some extended systems of more than one equation are separable, but not all such systems have been determined.

A possible connection between (A), (B), (C) is a source of further separable systems. Contraction of an extended equation with respect to some or all of its variables amounts to equating the values of these variables as given by the solution of the uncontracted equations. If the equations thus adjoined to the original equation are separable, the contracted equation is separable. In particular, if the adjoined equations are as in one or all of (A), (B), (C), the contracted equation is separable. A given extended equation when totally expanded thus generates a class of separable equations by contraction. At any stage of the process, binding equations may be imposed on some of the free parameters in the solution. The totality of equations in a class is determinable for a given extended equation. This classification cuts across the degree and 
the number of independent variables in resolvable equations. For example, it will be seen that

$$
x_{1} y_{1} z_{1}+\cdots+x_{n} y_{n} z_{n}=0,
$$

in $3 n$ independent variables $x_{j}, y_{j}, z_{j}$, and

$$
m_{1} u_{1}^{2}+\cdots+m_{n-1} u_{n-1}^{2}=m_{n} v_{1} \cdots v_{s},
$$

in $n+s-1$ independent variables $u_{i}, v_{j}$, with $m_{1}, \cdots, m_{n-1}$ arbitrary constants, are in the same class.

\section{Applications}

7. Totally expanded type. The totally expanded type of the general extended equation is

$$
\sum_{i=1}^{n} m_{i} x_{i 1} \cdots x_{i s_{i}^{\prime}}=0
$$

in which the $m_{i}$ are constants not equal to $0, n>2, s_{i}>1$, and the $x_{i j}$ are $s_{1}+\cdots+s_{n}$ independent variables.

In solving (7.1), four further general types appear:

$$
\xi_{1} \eta_{1}+\cdots+\xi_{n} \eta_{n}=0
$$

in which $\xi_{i}, \eta_{i}, i=1, \cdots, n$, are $2 n$ independent, variables;

$$
\theta_{1} \phi_{1}=\theta_{2} \phi_{2}=\cdots=\theta_{n} \phi_{n},
$$

in $2 n$ independent variables $\theta_{i}, \phi_{i}$;

$$
u u_{i}=v_{i} w_{i},
$$$$
i=1, \cdots, n \text {, }
$$

in which the $u, u_{i}, v_{i}, w_{i}$ are $3 n+1$ independent variables;

$$
u u_{i}=m_{i} w_{i},
$$$$
i=1, \cdots, n \text {, }
$$

in which the $m_{i}$ are $n$ constants not equal to 0 , and the $u, u_{i}, w_{i}$ are $2 n+1$ inde- $\cdot$ pendent variables. These four will be considered first.

The solution [3, p. $20(13)]$ of $(7.2)$ is

$$
\xi_{i}=\alpha \alpha_{i}, \quad \eta_{i}=-\sum_{j=1}^{j-1} \alpha_{j} \beta_{j, i}+\sum_{j=1}^{n-j} \alpha_{i+j} \beta_{i, i+j}
$$

for $i=1, \cdots, n$, with the convention that a sum in which the lower limit exceeds the upper is vacuous. The $\left(n^{2}+n+2\right) / 2$ parameters $\alpha, \alpha_{i}, \beta_{j, k}$ are free. If some of the signs in (7.5) are changed, the solution of the modified equation is obtained by making the corresponding changes in the values of either $\xi_{i}$ or $\eta_{i}$ in (7.6).

The resolution of (7.3) is recursive, the first step being the resolution of the $n-1$ equations 


$$
\theta_{i} \phi_{i}=\theta_{n} \phi_{n}, \quad i=1, \cdots, n-1 .
$$

The solution of the typical equation is

$$
\theta_{i}=\lambda_{i 1} \lambda_{i 2}, \quad \phi_{i}=\lambda_{i 3} \lambda_{i 4}, \quad \theta_{n}=\lambda_{i 1} \lambda_{i 4}, \quad \phi_{n}=\lambda_{i 3} \lambda_{i 2},
$$

in 4 free parameters $\lambda_{i j}$. In the second step the values of $\theta_{n}$ are equated, also those of $\phi_{n}$. The resulting systems are of the general type (7.3) with $n-1$ in place of $n$. By repetitions of the process the solution of (7.3), involving $(n) \equiv 2^{n}$ free parameters $\lambda_{1}, \cdots, \lambda_{(n)}$, is obtained in the form

$$
\theta_{i}=\Theta_{i}\left(\lambda_{1}, \cdots, \lambda_{(n)}\right), \quad \phi_{i}=\Phi_{i}\left(\lambda_{1}, \cdots, \lambda_{(n)}\right),
$$

in which each of $\Theta_{i}, \Phi_{i}$ is a monomial in $(n) / 2$ of the parameters $\lambda_{1}, \cdots, \lambda_{(n)}$, each occurring only once in a particular $\Theta_{i}$ or $\Phi_{i}$, and

$$
\Theta_{i}\left(\lambda_{1}, \cdots, \lambda_{(n)}\right) \Phi_{i}\left(\lambda_{1}, \cdots, \lambda_{(n)}\right)=\lambda_{1} \cdots \lambda_{(n)},
$$

for $i=1, \cdots, n$.

The solution of the typical equation in (7.4) is

$$
u=\theta_{i} \phi_{i}, \quad u_{i}=\psi_{i} \chi_{i}, \quad v_{i}=\theta_{i} \chi_{i}, \quad w_{i}=\psi_{i} \phi_{i},
$$

in 4 free parameters. The result of equating the values of $u$ for $i=1, \cdots, n$ is (7.3). The solution of (7.4) therefore follows from (7.7),

$$
\begin{aligned}
u & =\lambda_{1} \cdots \lambda_{(n)}, & u_{i} & =\psi_{i} \chi_{i}, \\
v_{i} & =\Theta_{i}\left(\lambda_{1}, \cdots, \lambda_{(n)}\right) \chi_{i}, & w_{i} & =\Phi_{i}\left(\lambda_{1}, \cdots, \lambda_{(n)}\right) \psi_{i},
\end{aligned}
$$

for $i=1, \cdots, n$ in $2^{n}+2 n$ free parameters $\lambda_{j}, \psi_{i}, \chi_{i}$.

The solution of (7.5) is obtained from (7.8) by $v_{i} \rightarrow m_{i}$, so that now the $\chi_{i}$ and those of the $\lambda_{j}$ occurring in the $\Theta_{i}\left(\lambda_{1}, \cdots, \lambda_{(n)}\right), i=1, \cdots, n$, are bound parameters.

The resolution of (7.1) now follows from that of (7.2) by

$$
m_{i} x_{i 2} \cdots x_{i s_{i}} \rightarrow \xi_{i}, \quad x_{i 1} \rightarrow \eta_{i},
$$

the first of which, by the first of (7.6), gives

$$
\alpha \alpha_{i}=m_{i} x_{i 2} \cdots x_{i s_{i}}, \quad i=1, \cdots, n .
$$

The resolution of this, by (7.5), (7.8), is reduced to that of the simple system

$$
\begin{gathered}
\Theta_{i}\left(\lambda_{1}, \cdots, \lambda_{(n)}\right) \chi_{i}=m_{i}, \\
x_{i 2} \cdots x_{i s_{i}}=\Phi_{i}\left(\lambda_{1}, \cdots, \lambda_{(n)}\right) \psi_{i},
\end{gathered}
$$

for $i=1, \cdots, n$. The first $n$ equations in (7.9) are the binding equations from (7.5). The values of the $\lambda_{j}, \chi_{i}, \psi_{i}$ in the solution of (7.9) are substituted into

$$
\alpha=\lambda_{1} \cdots \lambda_{(n)}, \quad \alpha_{i}=\psi_{i} \chi_{i} ;
$$

and these values of $\alpha, \alpha_{i}$ substituted into the second of (7.6) give 


$$
x_{i 1}=-\sum_{j=1}^{n-1} \alpha_{j} \beta_{j, i}+\sum_{j=1}^{n-i} \alpha_{i+j, \beta_{i, i+j}}
$$

The values of $x_{i 2}, \cdots, x_{i_{i}}$ in the solution of the second of (7.9) and (7.101) give the solution of (7.1).

Explicit formulas for the monomials $\Theta_{i}, \Phi_{i}$ can be given. They are complicated and will not be required. The second set of equations in (7.9) is solved first. The solution of the first equation, $i=1$, of the set gives the values of those of the $\lambda_{1}, \cdots, \lambda_{(n)}$ occurring in $\Phi_{1}$ as monomials in new parameters, $\mu_{1}, \mu_{2}, \cdots$. These values are substituted into $\Phi_{2}, \cdots, \Phi_{n}$, and the second equation of the set then gives $\mu_{1}, \mu_{2}, \ldots$ as monomials in new parameters $\sigma_{1}, \sigma_{2}, \cdots$, and so on, till $i=n$. Finally the values of the $\lambda$ 's determined as monomials in all the parameters thus introduced are substituted into the binding equations in (7.9), which are solved as in $\$ 3$.

8. Contracted type. The general contracted type derived from (7.1) is

$$
\sum_{i=1}^{n} m_{i} X_{i 1} \cdots X_{i t_{i}}=0
$$

in which $X_{i 1}, \cdots, X_{i t_{i}}$ is any contraction of $x_{i 1}, \cdots, x_{i s_{i}}$, so that the $X_{i 1}, \cdots, X_{i t_{i}}, i=1, \cdots, n$, are $t_{1}+\cdots+t_{n}$ independent monomials. At least two subtypes of (8.1) are separable.

In the first, in at least one of the monomials in each product $X_{i 1} \cdots X_{i t_{i}}$, $i=1, \cdots, n$, at least one of the independent variables occurs only to the first power; say $X_{i 1}=x_{i 1} X_{i 1}^{\prime}$, where $x_{i 1}, X_{i 1}^{\prime}$ are independent. The equation is then

$$
\sum_{i=1}^{n} m_{i} x_{i 1} X_{i 1}^{\prime} X_{i 2} \cdots X_{i t_{i}}=0,
$$

which is the general extended multiplicative equation defined in $\S 1$. It is reduced to (7.2) by

$$
m_{i} X_{i 1}^{\prime} X_{i 2} \cdots X_{i t_{i}} \rightarrow \xi_{i}, \quad x_{i 1} \rightarrow \eta_{i},
$$

and therefore, by (7.6), the associated simple system is

$$
\alpha \alpha_{i}=X_{i 1}^{\prime} X_{i 2} \cdots X_{i t_{i}}, \quad i=1, \cdots, n .
$$

The typical equation in (8.3) is of the general type

$$
u v=w_{1} \cdots w_{t},
$$

a simple equation whose solution may be given explicitly in terms of $2 t$ free parameters. The resolution of (8.3) is then similar to that of (7.5), when the $X_{i 1}^{\prime}, X_{i 2}, \cdots, X_{i t_{i}}$ are regarded as independent variables. In the solution the $X_{i 1}^{\prime}, \cdots$ are replaced by their expressions as monomials in the independent variables $x_{i j}$. The result is a simple system in these variables. 
A second separable type of (8.1) follows from the first on further contraction, but with respect to $x_{i 1}$ and another variable in the term in which $x_{i 1}$ occurs, for some or all of the terms in (8.2). The resolution of the equation thus contracted is reduced to that of the system of equations obtained from the solution of (8.2) by equating therein the values of the contracted variables. If the resulting system of equations is linear in some set of the free parameters in the solution of (8.2), the contracted equation is separable.

The cases of greatest interest are those in which the linear system has nontrivial solutions. If the linear system is $s$ homogeneous equations in $s$ unknowns, or if it is overconditioned, it gives merely a set of necessary conditions for the resolvability of the contracted equation. This is the case, for example, for homogeneous equations of the second degree derived from (7.1) with $s_{i}=2, i=1, \cdots, n$, by total contraction of each term.

9. Equal sums of squares. The solution of

$$
\sum_{i=1}^{n} x_{i}^{2}=\sum_{i=1}^{n} y_{i}^{2}
$$

is immediate from (7.2), (7.6) by

$$
\begin{aligned}
\xi_{i} & \rightarrow x_{i}+y_{i}, \quad \eta_{i} \rightarrow x_{i}-y_{i}: \\
2 x_{i} & =-\sum_{j=1}^{i-1} \alpha_{j} \beta_{j, i}+\alpha \alpha_{i}+\sum_{j=1}^{n-i} \alpha_{i+j} \beta_{i, i+j}, \\
2 y_{i} & =\sum_{j=1}^{i-1} \alpha_{j} \beta_{i, i}+\alpha \alpha_{i}-\sum_{j=1}^{n-i} \alpha_{i+j} \beta_{i, i+j} .
\end{aligned}
$$

By (9.1), (9.2) the resolution of

$$
\sum_{i=1}^{n} x_{i}^{2}=\sum_{i=1}^{n-1} y_{i}^{2}
$$

is reduced to that of

$$
\sum_{j=1}^{n-1} \alpha_{j} \beta_{j, n}+\alpha \alpha_{n}=0
$$

whose solution may be written down by a change of notation from (7.6). The solution of (9.3) then follows by substitution into (9.2).

Similarly, the resolution of

$$
x_{1}^{2}+x_{2}^{2}+\cdots+x_{n}^{2}=y^{2}
$$

is reduced to that of

$$
\sum_{j=1}^{i-1} \beta_{j, i} \alpha_{i}+\alpha \alpha_{i}-\sum_{j=1}^{n-i} \beta_{i, i+j} \alpha_{i+j}=0, \quad i=1, \cdots, n-1,
$$


a system of $n-1$ homogeneous linear diophantine equations in $n$ unknowns $\alpha, \alpha_{1}, \cdots, \alpha_{n-1}$. Hence (9.4) is separable.

Explicit formulas for the solution of (9.4) may be given. Let $\beta_{i}$ be the determinant (of order $n-1$ ) obtained by deleting the $j$ th column from the matrix ( $n-1$ rows, $n$ columns) of the system. The principal diagonal of $\beta_{n}$ is $\alpha, \alpha, \cdots, \alpha$, and the determinant is skew symmetric about this diagonal. It follows from Cayley's diagonal expansion for a determinant of this type that $\alpha=0$ and $\beta_{n}=0$ are compatible only if all the elements of $\beta_{n}$ vanish. This possibility is included as the trivial case $\rho=0$ in the solution below; otherwise, $\beta_{n} \neq 0$. Hence if $\rho$ is any integer multiple of the reciprocal of the G.C.D. of $\beta_{1}, \cdots, \beta_{n}$,

$$
\text { - } \alpha_{j}=\rho(-1)^{j-1} \beta_{j}, \quad j=1, \cdots, n .
$$

The solution of (9.4) is now written down from (9.2) by substituting these values of $\alpha_{j}$ into $x_{1}, \cdots, x_{n}$ and into $y_{n} \equiv y$.

From this solution that of

$$
x_{1}^{2}+\cdots+x_{n-1}^{2}=u v
$$

follows by $u \rightarrow y+x_{n}, v \rightarrow y-x_{n}$. More generally, as in solving (9.4), it is seen that

$$
x_{1}^{2}+\cdots+x_{n-s}^{2}=u_{1} v_{1}+\cdots+u_{s} v_{s}
$$

is separable. By $u_{j} \rightarrow y_{j}+x_{j}, v_{j} \rightarrow y_{j}-x_{j}$, it follows that

$$
x_{1}^{2}+\cdots+x_{n}^{2}=y_{1}^{2}+\cdots+y_{s}^{2}
$$

is separable. But when $s \neq 1, n-1$, there are not explicit formulas for the solution.

10. Equal sums of squares and elementary monomials. The type

$$
x_{1}^{2}+\cdots+x_{n-s}^{2}=u_{1} w_{1}+\cdots+u_{s} w_{s},
$$

in which $x_{1}, \cdots, x_{n-s}, u_{1}, \cdots, u_{s}$ are $n$ independent variables, $w_{1}, \cdots, w_{s}$ are $s$ independent monomials in any number of variables, and all the variables in (10.1) are independent, is a generalization of (9.6). It is separable.

Each term on the left is first totally expanded, $x_{i} \rightarrow x_{i} y_{i}$, and the $w_{j}$ are replaced by variables $v_{j}$ so that the $2 n$ variables in

$$
x_{1} y_{1}+\cdots+x_{n-s} y_{n-s}-v_{1} u_{1}-\cdots-v_{s} u_{s}=0
$$

are independent. By (7.6) the solution of this equation is

$$
\begin{array}{rrr}
x_{i}=\alpha \alpha_{i}, & y_{i}=-\sum_{j=1}^{i-1} \alpha_{j} \beta_{i, i}+\sum_{j=1}^{n-i^{\prime}} \alpha_{i+j} \beta_{i, i+j}, & i=1, \cdots, n-s ; \\
v_{k}=\alpha \alpha_{k}, & u_{k}=-\sum_{j=1}^{k-1} \alpha_{j} \beta_{i, k}+\sum_{j=1}^{n-k} \alpha_{k+j} \beta_{k, k+j}, & k=1, \cdots, s .
\end{array}
$$


Contraction with respect to $x_{i}, y_{i}$,

$$
-\sum_{j=1}^{i-1} \alpha_{j} \beta_{j, i}-\alpha \alpha_{i}+\sum_{j=1}^{n-i} \alpha_{i+j} \beta_{i, i+j}=0, \quad i=1, \cdots, n-s,
$$

and the substitution

$$
\alpha \alpha_{k}=w_{k}, \quad k=1, \cdots, s,
$$

reduce the resolution of (10.1) to that of the linear system (10.2) in $\alpha_{1}, \cdots, \alpha_{n}$ and the simple system (10.3). Hence (10.1) is separable. If $s=1$, explicit formulas for the solution may be given, as for (9.4), when (10.3) is solved explicitly for specific $w_{1}, \cdots, w_{k}$.

11. Generalization of $\S \S 9,10$. By $s_{i}=2, x_{i 1} \rightarrow x_{i}, y_{i 1} \rightarrow y_{i},(7.1)$ becomes

$$
m_{1} x_{1} y_{1}+\cdots+m_{n} x_{n} y_{n}=0,
$$

with arbitrary integer coefficients $m_{i}$. The cases $m_{i}=1, i=1, \cdots, n$, and its contractions treated in $\S 9$ are included in (11.1), and their solutions can be obtained by properly specializing that of (11.1). But the argument for (9.4) is inapplicable to the corresponding step in the resolution of (11.1), as the elements in the principal diagonal of the determinant concerned are not necessarily all positive or all negative; and the greater simplicity of the special case, also its historical interest, justify the separate treatment in $\$ 9$.

By $\xi_{i} \rightarrow u_{i} v_{i}, \eta_{i} \rightarrow w_{i},(7.2)$ becomes

$$
u_{1} v_{1} w_{1}+\cdots+u_{n} v_{n} w_{n}=0,
$$

and hence, from (7.6), for $i=1, \cdots, n$,

$$
\begin{aligned}
\alpha \alpha_{i} & =u_{i} v_{i}, \\
w_{i} & =-\sum_{j=1}^{i-1} \alpha_{j} \beta_{j, i}+\sum_{j=1}^{n-i} \alpha_{i+j} \beta_{i, i+j} .
\end{aligned}
$$

The solution of (11.1) will follow from these by $u_{i} \rightarrow m_{i}, v_{i} \rightarrow x_{i}, w_{i} \rightarrow y_{i}$.

The solution of the typical equation (11.2) is written in the form

$$
\alpha=a_{i} b_{i}, \quad \alpha_{i}=c_{i} \pi_{i}, \quad u_{i}=c_{i} a_{i}, \quad v_{i}=b_{i} \pi_{i} .
$$

Hence the $a_{i}, b_{i}$ are given by the simple system

$$
(\alpha=) a_{1} b_{1}=\cdots=a_{n} b_{n},
$$

which is of the type (7.3). The solution of (11.4) gives each of $a_{i}, b_{i}$ as a monomial in $2^{n-1}$ distinct free parameters, and for each $i$ the monomials $a_{i}, b_{2}$ are independent.

With the $a_{i}, b_{i}$ as determined by (11.4), the solution of (11.1) is thus

$$
x_{i}=b_{i} \pi_{i}, \quad y_{i}=-\sum_{j=1}^{i-1} c_{j} \beta_{j, i} \pi_{j}+\sum_{j=1}^{n-i} c_{i+j} \beta_{i, i+j} \pi_{i+j},
$$


with the binding equations

$$
c_{i} a_{i}=m_{i}, \quad i=1, \cdots, n .
$$

The free parameters are the $\pi^{\prime}$ s and $\beta^{\prime}$ s, in all $n(n+1) / 2$, and there are $2^{n}+n$ bound parameters in the $b_{i}, c_{i}$ determined by (11.4), (11.6).

The solution of

$$
\sum_{i=1}^{n} m_{i} x_{i}^{2}=\sum_{i=1}^{n} m_{i} y_{i}^{2}
$$

follows from $x_{i} \rightarrow x_{i}+y_{i}, y_{i} \rightarrow x_{i}-y_{i}$ applied to (11.5).

The solution of

$$
\sum_{i=1}^{n} m_{i} x_{i}^{2}=\sum_{i=1}^{n-1} m_{i} y_{i}^{2}
$$

is obtained from that of (11.7) by substituting the $\beta_{i, n}, \pi_{i}$ determined by

$$
\sum_{j=1}^{n-1} c_{j} \beta_{j, n} \pi_{j}=0
$$

which is of the general type (11.1), into the solution of (11.7).

If $n=2$,

$$
m_{1} x_{1}^{2}+\cdots+m_{n-1} x_{n-1}^{2}+m_{n} x_{n} y_{n}=0
$$

is a simple equation. Let $n>2$. The solution of (11.9) or, what is equivalent by $x_{n} \rightarrow x_{n}+y, y_{n} \rightarrow x_{n}-y$, that of

$$
m_{1} x_{1}^{2}+\cdots+m_{n} x_{n}^{2}=y^{2}
$$

falls into two parts. Contraction of (11.1) with respect to $x_{i}, y_{i}, i=1, \cdots$, $n-1$, gives (11.9), and hence, by (11.5),

$$
-\sum_{j=1}^{i-1} c_{j} \beta_{j, i} \pi_{j}-b_{i} \pi_{i}+\sum_{j=1}^{n-i} c_{i+j} \beta_{i, i+j} \pi_{i+j}=0, \quad i=1, \cdots, n-1,
$$

a system of $n-1$ linear homogeneous equations in $\pi_{1}, \cdots, \pi_{n}$.

If the values assigned to the $\beta$ 's (which are free parameters) are such that not all determinants of order $n-1$ in the matrix of the system (11.11) vanish, the $\beta$ 's and the system are regular, in the contrary case, singular, and likewise for the corresponding solutions.

In the regular case let $m$ be an arbitrary integer multiple of the reciprocal of the G.C.D. (for specific $\beta$ 's) of the $n$ determinants of order $n-1$ in the matrix, and let $\beta_{i}$ be the determinant obtained by deleting the $i$ th column of the matrix. Then

$$
\pi_{i}=(-1)^{i-1} m \beta_{i},
$$$$
i=1, \cdots, n \text {; }
$$ 
and by (11.5) the regular solution of (11.9) is

$$
\begin{aligned}
& x_{i}=(-1)^{i-1} m b_{i} \beta_{i}, \quad i=1, \cdots, n, \\
& y_{n}=-m \sum_{j=1}^{n-1}(-1)^{i-1} c_{j} \beta_{j} \beta_{j, n} .
\end{aligned}
$$

In the singular case, let $\theta_{1}, \cdots, \theta_{n}$ be integer parameters, restricted for the moment so that the vector $\left(\theta_{1}, \cdots, \theta_{n}\right)$ is not equal to any row vector of the matrix of (11.11) in the singular case. Then the determinant of the system formed by adjoining

$$
\theta_{1} \pi_{1}+\cdots+\theta_{n} \pi_{n}=0
$$

to (11.11) vanishes, and hence the augmented system has a non-trivial solution. Let $m^{\prime}$ be any integral multiple of the reciprocal of the G.C.D. of the determinants $\beta_{\text {? }}^{\prime}$ of the matrix of the system formed by (11.13) (as the first equation) and the $n-1$ equations obtained by omitting the last equation in (11.11), Then

$$
\pi_{i}=(-1)^{i-1} m^{\prime} \beta_{i}^{\prime}, \quad i=1, \cdots, n,
$$

and the singular solution is written down from (11.12) by the change in notation $m \rightarrow m^{\prime}, \beta_{i} \rightarrow \beta_{i}^{\prime}$. The restriction on $\left(\theta_{1}, \cdots, \theta_{n}\right)$ may be removed, as it excludes only the trivial solution, so that $\theta_{1}, \cdots, \theta_{n}$ in the singular solution are $n$ distinct free parameters.

The discussion for

$$
a_{1} x_{1}^{2}+\cdots+a_{n} x_{n}^{2}=b_{1} y_{1}^{2}+\cdots+b_{s} y_{s}^{2}
$$

parallels that of (9.7), and may be omitted. Likewise for (10.1) and

$$
a_{1} x_{1}^{2}+\cdots+a_{n-8} x_{n-s}^{2}=b_{1} u_{1} w_{1}+\cdots+b_{s} u_{s} w_{s}
$$

with the $a$ 's, $b$ 's arbitrary constants and the rest of the notation as in (10.1).

By the classical rational reduction of the general quadratic form $Q\left(x_{1}, \cdots, x_{t}\right)$ with integer coefficients to a sum of squares with rational number coefficients, it follows from (11.15) that

$$
Q\left(x_{1}, \cdots, x_{n-s}\right)=b_{1} u_{1} w_{1}+\cdots+b_{s} u_{s} w_{s}
$$

is separable. This includes all the preceding results (and an infinity more).

For general $n$ the equations in $\S \S 7-11$ are irreducible, as reducibility is defined in $\S 4$. They are also irreducible in the usual sense in any ring.

12. Example for $\S 11$. The unavoidable calculations for a given $n$ are concentrated in (11.4). It will be sufficient to indicate the details for $n=3$. In that case (11.1) is

$$
m_{1} x_{1} y_{1}+m_{2} x_{2} y_{2}+m_{3} x_{3} y_{3}=0,
$$

and the corresponding solution of (11.4) is 


$$
\begin{array}{lll}
a_{1}=\gamma_{1} \gamma_{2} \delta_{3} \delta_{4}, & a_{2}=\gamma_{1} \gamma_{4} \delta_{2} \delta_{3}, & a_{3}=\gamma_{1} \gamma_{2} \gamma_{3} \gamma_{4}, \\
b_{1}=\gamma_{3} \gamma_{4} \delta_{1} \delta_{2}, & b_{2}=\gamma_{2} \gamma_{3} \delta_{1} \delta_{4}, & b_{3}=\delta_{1} \delta_{2} \delta_{3} \delta_{4} .
\end{array}
$$

From the general discussion, the solution is

$$
\begin{array}{ll}
x_{1}=b_{1} \pi_{1}, & y_{1}=c_{2} \beta_{1,2} \pi_{2}+c_{3} \beta_{1,3} \pi_{3}, \\
x_{2}=b_{2} \pi_{2}, & y_{2}=-c_{1} \beta_{1,2} \pi_{1}+c_{3} \beta_{2,3} \pi_{3}, \\
x_{3}=b_{3} \pi_{3}, & y_{3}=-c_{1} \beta_{1,3} \pi_{1}-c_{2} \beta_{2,3} \pi_{2},
\end{array}
$$

with the binding equations

$$
m_{1}=c_{1} a_{1}, \quad m_{2}=c_{2} a_{2}, \quad m_{3}=c_{3} a_{3} .
$$

By $\$ 3$ the binding equations have solutions and are resolvable for any given $m_{1}, m_{2}, m_{3}$.

From the above solution those of the several contractions of the original equation are readily obtained by following the directions for the general case. As pointed out, the solution of the totally contracted equation

$$
m_{1} x_{1}^{2}+m_{2} x_{2}^{2}+m_{3} x_{3}^{2}=0
$$

is not obtainable by this method. The equations of total contraction will have non-trivial solutions $\pi_{1}, \pi_{2}, \pi_{3}$ if and only if

$$
b_{1} c_{2} c_{3} \beta_{2,3}^{2}+b_{2} c_{1} c_{3} \beta_{1,3}^{2}+b_{3} c_{1} c_{2} \beta_{1,2}^{2}=-b_{1} b_{2} b_{3}
$$

has solutions $\beta_{2,3}, \beta_{1,3}, \beta_{1,2}$, or, what is equivalent, if and only if $-\alpha^{2}$, where $\alpha \equiv \gamma_{1} \gamma_{2} \gamma_{3} \gamma_{4} \delta_{1} \dot{\delta}_{2} \delta_{3} \delta_{4}$, is represented in the form

$$
m_{1} m_{2} \beta_{1,2}^{2}+m_{2} m_{3} \beta_{2,3}^{2}+m_{1} m_{3} \beta_{1,3}^{2} .
$$

When this condition is satisfied, the solutions of the totally contracted equation are found from those of the totally expanded equation by substituting therein the values of $\pi_{1}, \pi_{2}, \pi_{3}$ determined by

$$
\begin{aligned}
& -b_{1} \pi_{1}+c_{2} \beta_{1,2} \pi_{2}+c_{3} \beta_{1,3} \pi_{3}=0, \\
& -c_{1} \beta_{1,2} \pi_{1}-b_{2} \pi_{2}+c_{3} \beta_{2,3} \pi_{3}=0,
\end{aligned}
$$

for each set of values of $\beta_{1,2}, \beta_{2,3}, \beta_{1,3}$ representing $-\alpha^{2}$ in the above form.

On another occasion examples of separable equations and separable systems of higher degrees will be given. An example of a separable equation whose solution is immediate from the calculations of this section is

$$
a x y+b z w=c u^{3},
$$

$a, b, c$ arbitrary constants.

\section{REFERENCES}

1. E. T. Bell, Reciprocal arrays and diophantine analysis, Amer. J. Math. vol. 55 (1933) pp. 50-66. 
2. G. Libri, Exposition d'un principe generale qui renferme toute la thérie des nombres. Memorie della Reale Accademia della Scienze di Torino vol. 28 (1824) pp. 272-280; also, ibid. p. 254. The "general principle" was extensively applied in several later papers; this was its first appearance.

3. Th. Skolem, Diophantische Gleichungen, Ergebnisse der Mathematik und ihrer Grenzgebiete vol. 5 (1938) no. 4.

4. M. Ward, $A$ type of multiplicative diophantine systems, Amer. J. Math. vol. 55 (1933) pp. 67-76. The large $N(S)$ in $\$ 5$ is from this paper, where $N(S)$ is determined for certain types of $S$.

California Institute of Technology 